\title{
Clinical Analysis of Gemcitabine Combined with Tegafur Chemotherapy after Radical Surgery on Pancreatic Cancer
}

\author{
Wei Zheng, Xiang Yan, Xiaoxue Song* \\ Shaanxi Provincial People's Hospital, Xi'an 710068, China
}

\begin{abstract}
[Abstract] Objective: To study and analyze the clinical efficacy of gemcitabine combined with Tegafur chemotherapy after radical resection of pancreatic cancer. Methods: The subjects of the study were 200 patients who were admitted to the hospital from January 2018 to February 2021 requiring chemotherapy after radical resection of pancreatic cancer. According to the different treatment methods, they were divided into a experimental group (gemcitabine combined with Tegafur chemotherapy) and a control group (single gemcitabine chemotherapy), and the treatment efficacy of the two groups of patients was observed and compared. Results: Compared with the control group, patients in the experimental group had significantly better treatment efficacy, quality of life scores and post-treatment anxiety and depression scores. The difference between the groups was significant $(\mathrm{p}<0.05)$. Conclusion: Gemcitabine combined with Tegafur chemotherapy for patients requiring chemotherapy after radical resection of pancreatic cancer can significantly improve the treatment efficacy for the disease, improve the patient's quality of life, and ensure that the patient's emotional state during treatment is more positive.
\end{abstract}

Key words: Radical resection of pancreatic cancer; Gemcitabine; Tegafur; Chemotherapy; Clinical efficacy

Publication date: May, 2021; Publication online: 31 May, 2021

*Corresponding author: Xiaoxue Song, zhengwei03168@163.com

As a malignant tumor disease, pancreatic cancer has a relatively high degree of malignancy. The clinical manifestations of patients are mainly abdominal pain. The risk factors for the disease include diabetes and long-term history of smoking and drinking. At present, chemotherapy is mainly used to improve the patient's conditions in clinic, but chemotherapy can cause various complications, so the chemotherapy scheme needs to be optimized. Single-drug chemotherapy is often used clinically, and combination drugs are now being considered for chemotherapy ${ }^{[1]}$. The research content of this paper is the clinical efficacy of gemcitabine combined with Tegafur chemotherapy after radical resection of pancreatic cancer. The following results were obtained:

\section{General information and methods}

\subsection{General information}

In this study, our hospital selected 200 patients who needed chemotherapy after radical resection of pancreatic cancer and divided them into two groups by random number table method. In the 100 cases control group, the male to female ratio was $4: 6$, and the average age was $(55.9 \pm 6.4)$ years. The male to female ratio of the 100 patients in the experimental group was 5:5, and the average age was (57.4 \pm 8.1$)$ years. There was no significant difference in age and gender between the two groups of patients $(\mathrm{P}>0.05)$. All the enrolled patients were aware of the study, volunteered to participate in it, and signed an informed consent. The patients were mentally clear and sane, and could accurately answer relevant research questions raised by the researchers, with no other serious life-threatening complications except for this disease. The excluded patients were very resistant to this study, or had other serious life-threatening diseases or could not answer the questions accurately. Or the family members of the patients were resistant to this study and refused to participate in the study. Or the patients were 
transferred to other hospitals or dropped out directly during the study ${ }^{[2]}$. The study was officially launched after obtaining the permission of the hospital ethics committee.

\subsection{Treatment methods}

Patients in the control group received gemcitabine treatment alone and received intravenous infusion of gemcitabine (National Medicine Zhunzi H20030105), and the experimental group received Tegafur on top of the above basis, and intravenous infusion of Tegafur (National Medicine Zhunzi H20080802) was administered. The duration of a course of treatment was set to 8 days, and the treatment efficacy was compared after all patients received 4 courses of treatment.

\subsection{Observation items and indices}

\subsubsection{Assessment criteria for treatment efficacy}

(1) Markedly effective: Various adverse symptoms have basically disappeared and no serious complications have been observed; (2) Effective: Various adverse symptoms have been improved, and the complications have been significantly improved after symptomatic treatment; (3) Ineffective: Those who do not meet the above criteria.

\subsubsection{Quality of life assessment criteria}

In-house quality of life questionnaires were distributed to the patients to fill in anonymously; valid questionnaires were collected on site, and the quality of life scores were compiled. The questionnaire was divided into 4 major items, each accounting for 25 points, with a full score of 100 points. The higher the score, the higher the patient's quality of life.

\subsubsection{Anxiety and depression scoring criteria}

It was evaluated by the Anxiety and Depression Score Scale. The higher the score, the heavier the negative emotion of the patient.

\subsection{Statistical method}

For the data and information involved in this study, SPSS20.0 statistical software was used for analysis and processing.

\section{Results}

\subsection{Comparison on the treatment efficacy between the two groups of patients}

The statistical results showed that the treatment efficacy of the experimental group was higher than that of the control group, and there were significant differences between the groups $(\mathrm{P}<0.05)$, which was statistically significant. See Table 1 for details.

Table 1. Comparison on the Treatment Efficacy between the Two Groups of Patients (n, \%)

\begin{tabular}{ccccc}
\hline Group & Markedly Effective & Effective & Ineffective & Efficacy \\
\hline Experimental $(\mathrm{n}=100)$ & & & $95.0 \%(95 / 100)$ \\
Control $(\mathrm{n}=100)$ & 90 & 5 & 5 & $83.0 \%(83 / 100)$ \\
$\mathrm{X}^{2}$ & 73 & 10 & 17 & 8.524 \\
$\mathrm{P}$ & & & $\mathrm{P}<0.05$ \\
\hline
\end{tabular}

\subsection{Comparison on the life quality scorings between} the two groups of patients

The statistical results show that the scores of physical functions, emotional functions, social functions and mental health of the experimental group were significantly higher than those of the control group. There were significant differences between the groups $(\mathrm{P}<0.05)$, which was statistically significant. See Table 2 for details.

Table 2. Comparison on the Life Quality Scorings between the Two Groups of Patients ( $p t s, x \pm s$ )

\begin{tabular}{ccccc}
\hline Group & Physical Functions & Emotional Functions & Social Functions & Mental Health \\
\hline Experimental $\quad(\mathrm{n}=100)$ & $89.48 \pm 6.14$ & $93.38 \pm 5.56$ & $91.52 \pm 5.13$ & $92.38 \pm 5.11$ \\
Control $(\mathrm{n}=100)$ & $72.45 \pm 6.16$ & $84.86 \pm 0.62$ & $82.57 \pm 5.11$ & $81.06 \pm 4.12$ \\
$\mathrm{t}$ & 8.174 & 5.358 & 8.527 & 9.358 \\
$\mathrm{P}$ & $<0.05$ & $<0.05$ & $<0.05$ & $<0.05$ \\
\hline
\end{tabular}

2.3 Comparison on the anxiety and depression scores before and after treatment between the two groups of patients
The statistical results show that before treatment, the anxiety and depression scores of the two groups of patients were higher, and there was no significant difference between the 
groups $(\mathrm{P}>0.05)$. After respective therapies, the scores of the two groups were reduced, and the experimental group was significantly lower than the control group, there were significant differences between the groups $(\mathrm{P}<0.05)$, which were statistically significant. See Table 3 for details.

Table 3. Comparison on the anxiety and depression scores before and after treatment between the two groups of patients $(\mathrm{x} \pm \mathrm{s})$

\begin{tabular}{ccccc}
\hline Group & $\begin{array}{c}\text { Anxiety } \\
\text { (before treatment) }\end{array}$ & $\begin{array}{c}\text { Anxiety } \\
\text { (after treatment) }\end{array}$ & $\begin{array}{c}\text { Depression } \\
\text { (before treatment) }\end{array}$ & $\begin{array}{c}\text { Depression } \\
\text { (after treatment) }\end{array}$ \\
\hline Experimental $(\mathrm{n}=100)$ & $22.38 \pm 5.47$ & $15.82 \pm 4.14$ & $25.32 \pm 4.03$ & $16.38 \pm 4.54$ \\
Control $(\mathrm{n}=100)$ & $22.86 \pm 5.12$ & $18.55 \pm 4.45$ & $25.02 \pm 5.31$ & $19.86 \pm 5.12$ \\
$\mathrm{t}$ & 0.174 & 9.358 & 0.527 & 9.402 \\
$\mathrm{P}$ & $>0.05$ & $<0.05$ & $>0.05$ & $<0.05$ \\
\hline
\end{tabular}

\section{Discussion}

At present, the cause of pancreatic cancer has not been clearly studied clinically, and the possible influencing factors include pathological changes in the pancreatic duct, pancreatic vesicles and islets. After contracting the illness, patients will have different degrees of pain, accompanied by symptoms such as loss of appetite and overall weakness, and their normal daily life is severely affected ${ }^{[3]}$. Chemotherapy can effectively improve adverse symptoms, and patients have a high degree of acceptance of treatment. However, during the actual application of chemotherapy, the therapeutic effect is often affected by the disease and the patient's own factors. For example, when the patient is receiving treatment, the condition is often already very serious, the physique is poor, and the body's immunity is extremely low. Therefore, the risk of chemotherapy is higher $^{[4]}$.

In recent years, some scholars have found through research that the common influencing factors of pancreatic cancer include pancreatic islet shadow and cancer cell composition, etc. If the optimal treatment time is missed, it will aggravate the necrosis of pancreatic tissue and threaten the life of patients ${ }^{[5]}$. When only one drug is administered in chemotherapy, the disease progression can only be controlled, but the disease cannot be eradicated. Therefore, the combination of drugs to treat the disease has been considered clinically. The selected treatment drug needs to have a significant analgesic effect to ensure that the patient can relieve body pain in a short time after taking the drug ${ }^{[6]}$. In addition, the selected drugs also need to improve the patient's body immunity and prevent other diseases.

Tegafur is a compound chemotherapeutic drug with high targeting specificity. In addition to killing cancer cells quickly, it can also avoid damaging cells and tissues of the body $^{[7]}$. It has a very wide range of clinical applications and is highly recognized. It is often used for gastric cancer, head and neck tumors, colon cancer and pancreatic cancer that cannot be treated by surgery. The curative effect is very prominent. Moreover, the drug can effectively enhance the anti-cancer activity, and the toxicity to the body is lower. In addition, the drug is very convenient to administer ${ }^{[8]}$. When gemcitabine is combined with Tegafur chemotherapy, the advantages of the two drugs complement each other, and the therapeutic effect is more prominent. The results of this study showed that the treatment efficacy of patients in the experimental group who received combination chemotherapy was significantly higher than that of the control group. The quality of life scores were significantly higher than those of the control group. The anxiety and depression scores after treatment were significantly lower than those of the control group. The differences are prominent $(\mathrm{P}<0.05)$ and statistically significant. It has been proved that gemcitabine combined with Tegafur chemotherapy can achieve the expected clinical effect for patients who require chemotherapy after radical resection of pancreatic cancer. It has to be pointed out that as the combination chemotherapy program was only carried out on traditional conventional pancreatic cancer patient samples, and the treatment was aimed at most patients, but in actual applications, there are individual differences between patients, and conventional treatment is more focused on single treatment, therefore lacking systemicity. In addition, as the research was based on a small sample of data, the conclusions obtained may be lacking in persuasiveness. It is expected that large-sample clinical studies can be carried out 
again in the future to ensure the persuasiveness of the research conclusions.

In conclusion: the implementation of gemcitabine combined with Tegafur chemotherapy for patients who need chemotherapy after radical resection of pancreatic cancer can significantly improve the treatment efficacy for the disease, improve the patient's quality of life, and ensure that the patient's emotional state during treatment is more positive.

\section{References}

[1] Tang JX. Clinical analysis of gemcitabine combined tegafur chemotherapy after the endotheration of pancreatic cancer [J]. Systems Medicine, 2020, 5(3):132-134.

[2] Liu M, Ji XH. Clinical efficacy of gemcitabine combined with s-1 capsule for pancreatic cancer patients treated with radical resection $[\mathrm{J}]$. China Health Care Nutrition, 2018, 28(12):213.

[3] Chen DS, Wu GX, Tang LZ. Clinical observation of gemcitabine combined with s-1 capsule for pancreatic cancer patients treated with radical resection $[\mathrm{J}]$. The Practical Journal of Cancer, 2014, (1):78-80.

[4] Chen R, Wu SB, Su R et al. The effect of adjuvant gemcitabine plus gimeracil and oteracil porassium capsules chemotherapy after curative resection for pancreatic cancer $[\mathrm{J}]$. Chinese Journal of General Surgery, 2011, 20(3):217-221.

[5] Wang H. The effect of gemcitabine combined with S-1 capsule chemotherapy on the quality of life of patients after radical operation of pancreatic cancer [C]. Chinese Journal of Hypertension. Comprehensive journal of the national training course on hypertension prevention and control knowledge and the paper of healthy blood pressure China travel to Zhangzhou in Fujian Province. 2014.

[6] Chen H. Application effect of gemcitabine combined with tegafur chemotherapy in patients after radical resection of pancreatic cancer [J]. Chinese Journal of Modern Drug Application, 2019, 13(1):80-81.

[7] Zhu L, Huang Y. Application of gemcitabine plus tegafur chemotherapy in pancreatic cancer patients after radical surgery $[\mathrm{J}]$. Chinese Journal of Clinical Oncology and Rehabilitation, 2016, 23(11):1319-1321.

[8] Ke LH, Luo HQ, Hu XX et al. A comparative analysis of gemcitabine combined with s-1 or oxaliplatin as the first line chemotherapy for advanced pancreatic cancer [J]. Anti-Tumor Pharmacy, 2014, (6):441-445. 